\title{
A POINTWISE APPROXIMATION THEOREM FOR LINEAR COMBINATIONS OF BERNSTEIN POLYNOMIALS
}

\author{
SHUNSHENG GUO, SHUJIE YUE, CUIXIANG LI, GE YANG AND YIGUO SUN
}

Abstract. Recently, Z. Ditzian gave an interesting direct estimate for Bernstein polynomials. In this paper we give direct and inverse results of this type for linear combinations of Bernstein polynomials.

\section{IntroduCtion}

For the Bernstein polynomial

$$
B_{n}(f, x)=\sum_{k=0}^{n} f\left(\frac{k}{n}\right) p_{n k}(x), \quad p_{n k}(x)=\left(\begin{array}{l}
n \\
k
\end{array}\right) x^{k}(1-x)^{n-k},
$$

Berens and Lorentz showed in [1] that

$$
B_{n}(f, x)-f(x)=O\left(\left(\frac{1}{\sqrt{n}} \delta_{n}(x)\right)^{\alpha}\right) \Longleftrightarrow \omega^{1}(f, t)=O\left(t^{\alpha}\right),
$$

where $0<\alpha<1, \delta_{n}(x)=\varphi(x)+\frac{1}{\sqrt{n}}, \varphi(x)=\sqrt{x(1-x)}$.

Recently, Ditzian [3] gave the following interesting result.

$$
\left|B_{n}(f, x)-f(x)\right| \leq C \omega_{\varphi^{\lambda}}^{2}\left(f, n^{-\frac{1}{2}} \varphi(x)^{1-\lambda}\right), 0 \leq \lambda \leq 1 .
$$

However, Ditzian did not consider the inverse result in [3]. We did give such an inverse result in [6], where we obtained the following equivalence.

$$
B_{n}(f, x)-f(x)=O\left(\left(n^{-\frac{1}{2}} \varphi(x)^{1-\lambda}\right)^{\alpha}\right) \Longleftrightarrow \omega_{\varphi^{\lambda}}^{2}(f, t)=O\left(t^{\alpha}\right), 0<\alpha<2 .
$$

In this paper we consider linear combinations of Bernstein polynomials, that is

$$
B_{n, r}(f, x)=\sum_{i=0}^{r-1} C_{i}(n) B_{n_{i}}(f, x),
$$

1991 Mathematics Subject Classification. 41A25, 41A36, 41A17.

Key words and phrases. Pointwise approximation, Bernstein polynomial.

Received: July 2, 1996. 
where $n_{i}$ and $C_{i}$ satisfy [5]
(a) $n=n_{0}<n_{1}<\cdots<n_{r-1} \leq K n$;
(b) $\sum_{i=0}^{r-1}\left|C_{i}(n)\right|<C$
(c) $\sum_{i=0}^{r-1} C_{i}(n)=1$
(d) $\sum_{i=0}^{r-1} C_{i}(n) n_{i}^{-\rho}=0, \quad \rho=1,2, \cdots, r-1$.

We recall that

$$
\omega_{\varphi^{\lambda}}^{r}(f, t)=\sup _{0<h \leq t} \sup _{x \pm \frac{r}{2} h \varphi^{\lambda}(x) \in[0,1]}\left|\Delta_{h \varphi^{\lambda}(x)}^{r} f(x)\right|
$$

is equivalent to the $K$-functional

$$
K_{\varphi^{\lambda}}\left(f, t^{r}\right)=\inf _{g^{(r-1)} \in A . C_{l o c}}\left(\|f-g\|_{c[0,1]}+t^{r}\left\|\varphi^{r \lambda} g^{(r)}\right\|_{C[0,1]}\right) .
$$

We write $\omega_{\varphi^{\lambda}}^{r}(f, t) \sim K_{\varphi^{\lambda}}\left(f, t^{r}\right)$, i.e., there exists a constant $C$ such that

$$
C^{-1} K_{\varphi^{\lambda}}\left(f, t^{r}\right) \leq \omega_{\varphi^{\lambda}}^{r}(f, t) \leq C K_{\varphi^{\lambda}}\left(f, t^{r}\right) .
$$

Now we state our main result.

Theorem. For $f \in C[0,1], 0<\alpha<r, 0 \leq \lambda \leq 1$, we have

$$
B_{n, r}(f, x)-f(x)=O\left(\left(n^{-\frac{1}{2}} \delta_{n}^{1-\lambda}(x)\right)^{\alpha}\right) \Longleftrightarrow \omega_{\varphi^{\lambda}}^{r}(f, t)=O\left(t^{\alpha}\right) .
$$

Remark. In the case $r=1$ and $\lambda=0$ our result is (1.2) of Berens and Lorentz [1].

\section{Direct THEOREM}

In this section we prove the direct part of (1.8). We need the K-functional (see [5], p. 24):

$$
\bar{K}_{\varphi^{\lambda}}\left(f, t^{r}\right)=\inf _{g^{(r-1)} \in A . C_{l o c}}\left\{\|f-g\|+t^{r}\left\|\varphi^{r \lambda} g^{(r)}\right\|+t^{r /\left(1-\frac{\lambda}{2}\right)}\left\|g^{(r)}\right\|\right\} .
$$

which is also equivalent to $\omega_{\varphi^{\lambda}}^{r}(f, t)$.

Theorem 1. For $f \in C[0,1], 0 \leq \lambda \leq 1$,

$$
\left|B_{n, r}(f, x)-f(x)\right| \leq A \omega_{\varphi^{\lambda}}\left(f, n^{-\frac{1}{2}} \delta_{n}(x)^{1-\lambda}\right) .
$$

Proof. By (2.1), we may choose $g_{n} \equiv g_{n . x . \lambda}$ for a fixed $x$ and $\lambda$ such that

$$
\begin{gathered}
\left\|f-g_{n}\right\| \leq A_{1} \omega_{\varphi^{\lambda}}^{r}\left(f, n^{-\frac{1}{2}} \delta_{n}^{1-\lambda}(x)\right), \\
\left(n^{-\frac{1}{2}} \delta_{n}^{1-\lambda}(x)\right)^{r}\left\|\varphi^{r \lambda} g_{n}^{(r)}\right\| \leq A_{2} \omega_{\varphi^{\lambda}}^{r}\left(f, n^{-\frac{1}{2}} \delta_{n}^{1-\lambda}(x)\right), \\
\left(n^{-\frac{1}{2}} \delta_{n}^{1-\lambda}(x)\right)^{2 r /(2-\lambda)}\left\|g_{n}^{(r)}\right\| \leq A_{3} \omega_{\varphi^{\lambda}}^{r}\left(f, n^{-\frac{1}{2}} \delta_{n}^{1-\lambda}(x)\right) .
\end{gathered}
$$

We recall that $[5$, p. 134]

$$
B_{n, r}\left((\cdot-x)^{k}, x\right)=0, \quad k=1,2, \cdots, r-1
$$


and that $[5$, p. 138$]$

$$
B_{n}\left((\cdot-x)^{2 j}, x\right)=\sum_{m=0}^{j-1} \frac{\varphi(x)^{2(j-m)}}{n^{j-m}} n^{-2 m} q_{m}(x),
$$

where $q_{m}(x)$ are fixed bounded polynomials. Therefore

$$
B_{n}\left((\cdot-x)^{2 j}, x\right) \leq M n^{-j} \delta_{n}^{2 j}(x) .
$$

From the definition of the $B_{n, r}$ we have

$\left|B_{n, r}(f, x)-f(x)\right| \leq\left|B_{n, r}\left(f-g_{n}, x\right)\right|+\left|f(x)-g_{n}(x)\right|+\left|B_{n, r}\left(g_{n}, x\right)-g_{n}(x)\right|$

$$
\leq(C+1)\left\|f-g_{n}\right\|+\left|B_{n, r}\left(g_{n}, x\right)-g_{n}(x)\right| .
$$

As in [4, Lemma 5.3], we obtain

$$
\begin{aligned}
& \left|\int_{x}^{t}(t-u)^{r-1} g_{n}^{(r)}(u) d u\right| \leq\left|\frac{(t-x)^{r-1}}{\delta_{n}^{r \lambda}(x)} \int_{x}^{t} \delta_{n}^{r \lambda}(u) g_{n}^{(r)}(u) d u\right|, \\
& \left|\int_{x}^{t}(t-u)^{r-1} g_{n}^{(r)}(u) d u\right| \leq\left|\frac{(t-x)^{r-1}}{\varphi^{r \lambda}(x)} \int_{x}^{t} \varphi^{r \lambda}(u) g_{n}^{(r)}(u) d u\right| .
\end{aligned}
$$

Using (2.6), (2.7) and (2.9), we get

$$
\begin{aligned}
& \left|B_{n, r}\left(g_{n}, x\right)-g_{n}(x)\right| \\
& =\left|B_{n, r}\left(\frac{1}{(r-1) !} \int_{x}^{t}(t-u)^{r-1} g_{n}^{(r)}(u) d u, x\right)\right| \\
& \leq \sum_{i=0}^{r-1}\left|C_{i}(n)\right|\left\|\delta_{n}^{r \lambda} g_{n}^{(r)}\right\|_{\infty} \frac{1}{(r-1) !} B_{n_{i}}\left(\frac{|t-x|^{r}}{\delta_{n}^{r \lambda}(x)}, x\right) \\
& \leq \sum_{i=0}^{r-1}\left|C_{i}(n)\right|\left\|\delta_{n}^{r \lambda} g_{n}^{(r)}\right\|_{\infty} \delta_{n}^{-r \lambda}(x) M_{1} n^{-\frac{r}{2}} \delta_{n}^{r}(x) \\
& \leq C M_{1} n^{-\frac{r}{2}} \delta_{n}^{r(1-\lambda)}(x)\left\|\delta_{n}^{r \lambda} g_{n}^{(r)}\right\|_{\infty} .
\end{aligned}
$$

Similarly, by (2.10) we have

$$
\left|B_{n, r}\left(g_{n}, x\right)-g_{n}(x)\right| \leq C M_{1} n^{-\frac{r}{2}} \delta_{n}^{r}(x) \varphi^{-r \lambda}(x)\left\|\varphi^{r \lambda} g_{n}^{(r)}\right\| .
$$

If $x \in E_{n}=\left[\frac{1}{n}, 1-\frac{1}{n}\right]$, then $\delta_{n}(x) \sim \varphi(x)$, and, by (2.4) and (2.12),

$$
\begin{aligned}
\left|B_{n, r}\left(g_{n}, x\right)-g_{n}(x)\right| & \leq M_{2}\left(n^{-\frac{1}{2}} \delta_{n}^{1-\lambda}(x)\right)^{r}\left\|\varphi^{r \lambda} g_{n}^{(r)}\right\| \\
& \leq M_{2} A_{2} \omega_{\varphi^{\lambda}}^{r}\left(f, n^{-\frac{1}{2}} \delta_{n}^{1-\lambda}(x)\right) .
\end{aligned}
$$


400 SHUNSheng GUO, SHUJIE YUE, CUIXIANG LI, GE YANG AND YIGUO SUN

If $x \in E_{n}^{c}=\left[0, \frac{1}{n}\right) \cup\left(1-\frac{1}{n}, 1\right]$, then $\delta_{n}(x) \sim \frac{1}{\sqrt{n}}$, and by $(2.4),(2.5)$ and (2.11) we obtain

$$
\begin{aligned}
\left|B_{n, r}\left(g_{n}, x\right)-g_{n}(x)\right| \leq & M_{3}\left(n^{-\frac{1}{2}} \delta_{n}^{1-\lambda}(x)\right)^{r}\left(\left\|\varphi^{r \lambda} g_{n}^{(r)}\right\|+\left\|n^{-\frac{r \lambda}{2}} g_{n}^{(r)}\right\|\right) \\
\leq & M_{4}\left(\left(n^{-\frac{1}{2}} \delta_{n}^{1-\lambda}(x)\right)^{r}\left\|\varphi^{r \lambda} g_{n}^{(r)}\right\|\right. \\
& \left.+\left(n^{-\frac{1}{2}} \delta_{n}^{1-\lambda}(x)\right)^{2 r /(2-\lambda)}\left\|g_{n}^{(r)}\right\|\right) \\
\leq & M_{5} \omega_{\varphi^{\lambda}}^{r}\left(f, n^{-\frac{1}{2}} \delta_{n}^{1-\lambda}(x)\right) .
\end{aligned}
$$

From (2.3), (2.8), (2.13) and (2.14) we get (2.2).

\section{INVERSE THEOREM}

In this section we prove the inverse part of (1.8).

Theorem 2. For $f \in C[0,1], 0<\alpha<r, 0 \leq \lambda \leq 1$, if

$$
\left|B_{n, r}(f, x)-f(x)\right| \leq B\left(n^{-\frac{1}{2}} \delta_{n}^{1-\lambda}(x)\right)^{\alpha}
$$

then

$$
\omega_{\varphi^{\lambda}}^{r}(f, t)=O\left(t^{\alpha}\right)
$$

To prove Theorem 2 we need some new notation and some lemmas. We use the following notation.

$$
\begin{gathered}
C_{0}=\{f \in C[0,1], \quad f(0)=f(1)=0\}, \\
\|f\|_{0}=\sup _{x \in(0,1)}\left|\delta_{n}^{\alpha(\lambda-1)}(x) f(x)\right|, \\
C_{\lambda}^{0}=\left\{f \in C_{0},\|f\|_{0}<\infty\right\}, \\
\|f\|_{r}=\sup _{x \in(0,1)}\left|\delta_{n}^{r+\alpha(\lambda-1)}(x) f^{(r)}(x)\right|, \\
C_{\lambda}^{r}=\left\{f \in C_{0},\|f\|_{r}<\infty, f^{(r-1)} \in A . C_{l o c}\right\} .
\end{gathered}
$$

For $f \in C_{0}$ we define the $K$-functional as follows:

$$
K_{\lambda}^{\alpha}\left(f, t^{r}\right)=\inf _{g \in C_{\lambda}^{r}}\left\{\|f-g\|_{0}+t^{r}\|g\|_{r}\right\} .
$$

We also need the following lemmas which will be proved in the next section.

Lemma 3.1. If $n \in N, 0<\alpha<r$, then

$$
\left\|B_{n} f\right\|_{r} \leq B_{1} n^{\frac{r}{2}}\|f\|_{0} \quad\left(f \in C_{\lambda}^{0}\right) .
$$

and

$$
\left\|B_{n} f\right\|_{r} \leq B_{2}\|f\|_{r} \quad\left(f \in C_{\lambda}^{r}\right) .
$$

Lemma 3.2. For $0<t<\frac{1}{8 r}$, $\frac{r t}{2} \leq x \leq 1-\frac{r t}{2}$ and $0 \leq \beta \leq r$, we have

$$
\int_{-\frac{t}{2}}^{\frac{t}{2}} \cdots \int_{-\frac{t}{2}}^{\frac{t}{2}} \delta_{n}^{-\beta}\left(x+\sum_{k=1}^{r} u_{k}\right) d u_{1} \cdots d u_{r} \leq C(\beta) t^{r} \delta_{n}^{-\beta}(x) .
$$

Now we prove (3.1). 
Proof of (3.1). Since $B_{n}(f, x)$ preserves linear functions, we consider only $f \in C_{0}$ for $r>1$. If $r=1, f(x)=a x+b$ then $\omega_{\varphi^{\lambda}}^{1}(a x+b, t)=a \varphi^{\lambda}(x) t \leq$ $a t^{\alpha} \quad(0<\alpha<1)$.

So, we may assume $f \in C_{0}$. From (3.2) we have

$$
K_{\lambda}^{\alpha}\left(f, t^{r}\right) \leq\left\|B_{n, r}(f)-f\right\|_{0}+t^{r}\left\|B_{n, r} f\right\|_{r}
$$

and we may choose $g \in C_{\lambda}^{r}$ such that

$$
\|f-g\|_{0} \leq 2 K_{\lambda}^{\alpha}\left(f, n^{-\frac{1}{2}}\right) \quad \text { and } \quad n^{-\frac{r}{2}}\|g\|_{r} \leq 2 K_{\lambda}^{\alpha}\left(f, n^{-\frac{1}{2}}\right) .
$$

By the assumption of Theorem 2, one has

$$
\left\|B_{n, r}(f, x)-f(x)\right\|_{0} \leq B n^{-\frac{\alpha}{2}} .
$$

Using Lemma 3.1 and (3.7) we have

$$
\begin{aligned}
\left\|B_{n, r}(f)\right\|_{r} & \leq\left\|B_{n, r}(f-g)\right\|_{r}+\left\|B_{n, r} g\right\|_{r} \\
& \leq M\left(n^{\frac{r}{2}}\|f-g\|_{0}+\|g\|_{r}\right) \\
& \leq 2 M n^{\frac{r}{2}} K_{\lambda}^{\alpha}\left(f, n^{-\frac{r}{2}}\right) .
\end{aligned}
$$

From (3.6), (3.8) and (3.9) we obtain

$$
K_{\lambda}^{\alpha}\left(f, t^{r}\right) \leq M_{1}\left(n^{-\frac{\alpha}{2}}+t^{r} n^{\frac{r}{2}} K_{\lambda}^{\alpha}\left(f, n^{-\frac{r}{2}}\right)\right)
$$

and this implies, via the Berens-Lorentz lemma [1], that if $\alpha<r$ then

$$
K_{\lambda}^{\alpha}\left(f, t^{r}\right) \leq M_{2} t^{\alpha} .
$$

On the other hand, notice that $\delta_{n}^{\alpha(1-\lambda)}(x)$ is concave. So, we have, for $g \in C_{\lambda}^{r}$,

$$
\begin{aligned}
\left|\Delta_{t \varphi^{\lambda}(x)}^{r} g(x)\right| & \leq\|g\|_{0} \sum_{j=0}^{r}\left(\begin{array}{l}
r \\
j
\end{array}\right) \delta_{n}^{\alpha(1-\lambda)}\left(x+\left(j-\frac{r}{2}\right) t \varphi^{\lambda}(x)\right) \\
& \leq\|g\|_{0} 2^{r} \delta_{n}^{\alpha(1-\lambda)}(x) .
\end{aligned}
$$

Using Lemma 3.2 for $g \in C_{\lambda}^{r}, 0<t \varphi^{\lambda}(x)<\frac{1}{8 r}$ and $\frac{r t \varphi^{\lambda}(x)}{2} \leq x \leq 1-\frac{r t \varphi^{\lambda}(x)}{2}$, we have

$$
\begin{aligned}
& \left|\Delta_{t \varphi^{\lambda}(x)}^{r} g(x)\right| \\
& \leq\left|\int_{-\frac{t}{2} \varphi^{\lambda}(x)}^{\frac{t}{2} \varphi^{\lambda}(x)} \cdots \int_{-\frac{t}{2} \varphi^{\lambda}(x)}^{\frac{t}{2} \varphi^{\lambda}(x)} g^{(r)}\left(x+u_{1}+\cdots+u_{r}\right) d u_{1} \cdots d u_{r}\right| \\
& \leq\|g\|_{r} \int_{-\frac{t}{2} \varphi^{\lambda}(x)}^{\frac{t}{2} \varphi^{\lambda}(x)} \\
& \cdots \int_{-\frac{t}{2} \varphi^{\lambda}(x)}^{\frac{t}{2} \varphi^{\lambda}(x)} \delta_{n}^{-r+\alpha(1-\lambda)}\left(x+u_{1}+\cdots+u_{r}\right) d u_{1} \cdots d u_{r} \\
& \leq M_{3} t^{r} \varphi^{r \lambda}(x) \delta_{n}^{-r+\alpha(1-\lambda)}(x)\|g\|_{r} \leq M_{3} t^{r} \delta_{n}^{(\alpha-r)(1-\lambda)}(x)\|g\|_{r} .
\end{aligned}
$$


402 SHUNShENG GUO, SHUJIE YUE, CUIXIANG LI, GE YANG AND YIGUO SUN

From (3.9)-(3.12), $0<t \varphi^{\lambda}(x)<\frac{1}{8 r}, \frac{r t \varphi^{\lambda}(x)}{2} \leq x \leq 1-\frac{r t \varphi^{\lambda}(x)}{2}$ and by choosing an appropriate $g$, we have

$$
\begin{aligned}
\left|\Delta_{t \varphi^{\lambda}(x)}^{r} f(x)\right| & \leq\left|\Delta_{t \varphi^{\lambda}(x)}^{r}(f-g)(x)\right|+\left|\Delta_{t \varphi^{\lambda}(x)}^{r} g(x)\right| \\
& \leq M_{4} \delta_{n}^{\alpha(1-\lambda)}(x)\left\{\|f-g\|_{0}+t^{r} \delta_{n}^{r(\lambda-1)}(x)\|g\|_{r}\right\} \\
& \leq 2 M_{4} \delta_{n}^{\alpha(1-\lambda)} K_{\lambda}^{\alpha}\left(f, \frac{t^{r}}{\delta_{n}^{r(1-\lambda)}(x)}\right) \\
& \leq 2 M_{4} \delta_{n}^{\alpha(1-\lambda)}(x) \frac{t^{\alpha}}{\delta_{n}^{\alpha(1-\lambda)}(x)} \\
& =M_{5} t^{\alpha} .
\end{aligned}
$$

The proof of (3.1) is complete.

\section{Proofs of the Lemmas}

Proof of Lemma 3.1. We first prove (3.3). Suppose that $E_{n}=\left[\frac{1}{n}, 1-\frac{1}{n}\right]$. For $x \in E_{n}^{c}=\left(0, \frac{1}{n}\right) \cup\left(1-\frac{1}{n}, 1\right)$, we have [5] by Hölder's inequality

$$
\begin{aligned}
& \left|B_{n}^{(r)}(f, x)\right|=\left|\frac{n !}{(n-r) !} \sum_{k=0}^{n-r} \vec{\Delta}_{\frac{1}{n}}^{r} f\left(\frac{k}{n}\right) p_{n-r, k}(x)\right| \\
& \leq L n^{r}\|f\|_{0} \sum_{k=0}^{n-r} \sum_{j=0}^{r}\left(\begin{array}{l}
r \\
j
\end{array}\right) \delta_{n}^{\alpha(1-\lambda)}\left(\frac{k+r-j}{n}\right) p_{n-r, k}(x) \\
& \leq L_{1} n^{r}\|f\|_{0}\left(\sum_{k=0}^{n-r} \sum_{j=0}^{r} \delta_{n}^{2 r}\left(\frac{k+r-j}{n}\right) p_{n-r, k}(x)\right)^{\alpha(1-\lambda) / 2 r} .
\end{aligned}
$$

For $n>4 r$ we have

$$
\begin{aligned}
\sum_{k=0}^{n-r} \varphi^{2 r}\left(\frac{k+1}{n}\right) & p_{n-r, k}(x) \\
& =\left(\sum_{k=0}^{2 r}+\sum_{k=2 r+1}^{n-3 r}+\sum_{k=n-3 r+1}^{n-r}\right) \varphi^{2 r}\left(\frac{k+1}{n}\right) p_{n-r, k}(x) \\
& :=I_{1}+I_{2}+I_{3} .
\end{aligned}
$$

Obviously $I_{1}+I_{3} \leq 2((3 r+1) !)^{r} / n^{r}$ and, by simple computation, we have

$$
\begin{aligned}
I_{2}= & \sum_{k=2 r+1}^{n-3 r}\left(\frac{k+1}{n}\right)^{r}\left(\frac{n-k-1}{n}\right)^{r} \frac{(n-r) !}{(n-r-k) ! k !} x^{k}(1-x)^{n-r-k} \\
= & x^{r}(1-x)^{r} \sum_{k=2 r+1}^{n-3 r} \frac{(n-r) \cdots(n-3 r+1)}{n^{2 r}} \cdot \frac{(k+1)^{r}}{k \cdots(k-r+1)} \\
& \cdot \frac{(n-k-1)^{r}}{(n-r-k) \cdots(n-2 r-k+1)} p_{n-3 r, k-r}(x) \\
\leq & 2 \times 2^{r} \times 3^{r} \varphi^{2 r}(x) .
\end{aligned}
$$


By this and $\delta_{n}^{2 r}\left(\frac{k+r-j}{n}\right) \sim \varphi^{2 r}\left(\frac{k+1}{n}\right)+\left(\frac{1}{n}\right)^{r}$, one has

$$
\left|B_{n}^{(r)}(f, x)\right| \leq L_{2} n^{r}\|f\|_{0} \delta_{n}^{\alpha(1-\lambda)}(x) .
$$

Recalling that $x \in E_{n}^{c}$ implies $\delta_{n}(x) \sim \frac{1}{\sqrt{n}}$, we see that

$$
\delta_{n}^{r+\alpha(\lambda-1)}(x)\left|B_{n}^{(r)}(f, x)\right| \leq L_{3} n^{\frac{r}{2}}\|f\|_{0} .
$$

For $x \in E_{n}$ we use the expression (cf. [5])

$$
B_{n}^{(r)}(f, x)=(x(1-x))^{-r} \sum_{i=0}^{r} Q_{i}(x, n) n^{i} \sum_{k=0}^{n}\left(\frac{k}{n}-x\right)^{i} f\left(\frac{k}{n}\right) p_{n, k}(x)
$$

with $Q_{i}(n, x)$ a polynomials in $n x(1-x)$ of degree $[(r-i) / 2]$ with nonconstant bounded coefficients. Thus,

$$
\left|(x(1-x))^{-r} Q_{i}(x, n) n^{i}\right| \leq L_{4}\left(\frac{n}{x(1-x)}\right)^{(r+i) / 2} \quad \text { for } x \in E_{n} .
$$

If $x \in E_{n}$ then $\delta_{n}(x) \sim \varphi(x)$ and, recalling that $\delta_{n}^{2}\left(\frac{k}{n}\right) \sim \varphi^{2}\left(\frac{k}{n}\right)+\frac{1}{n}$, we have by using Hölder inequality twice

$$
\begin{aligned}
\left|B_{n}^{(r)}(f, x)\right| \leq & L_{5} \sum_{i=0}^{r}\left(\frac{n}{\varphi^{2}(x)}\right)^{(r+i) / 2} \sum_{k=0}^{n}\left|\frac{k}{n}-x\right|^{i} \delta_{n}^{\alpha(1-\lambda)}\left(\frac{k}{n}\right) p_{n, k}(x)\|f\|_{0} \\
\leq & L_{5}\|f\|_{0} \sum_{i=0}^{r}\left(\frac{n}{\varphi^{2}(x)}\right)^{(r+i) / 2}\left(\sum_{k=0}^{n}\left(\frac{k}{n}-x\right)^{2 i} p_{n, k}(x)\right)^{\frac{1}{2}} \\
& \left(\sum_{k=0}^{n}\left(\varphi^{2}\left(\frac{k}{n}\right)+\frac{1}{n}\right)^{r} p_{n, k}(x)\right)^{\frac{\alpha(1-\lambda)}{2 r}} .
\end{aligned}
$$

Proceeding as in (4.1), we obtain

$$
\sum_{k=0}^{n}\left(\varphi^{2}\left(\frac{k}{n}\right)+\frac{1}{n}\right)^{r} p_{n, k}(x) \leq C \delta_{n}^{2 r}(x) .
$$

It is known that for $m \in N[5]$

$$
\left|\sum_{k=0}^{n}\left(\frac{k}{n}-x\right)^{2 m} p_{n, k}(x)\right| \leq L_{6} n^{-m} \varphi^{2 m}(x) \quad \text { for } x \in E_{n} .
$$

Consequently, with $x \in E_{n}, \delta_{n}(x) \sim \varphi(x)$ and

$$
\begin{aligned}
\left|B_{n}^{(r)}(f, x)\right| & \leq L_{6}\|f\|_{0} \sum_{i=0}^{r}\left(\frac{n}{\varphi^{2}(x)}\right)^{(r+i) / 2}\left(\frac{\varphi^{2 i}(x)}{n^{i}}\right)^{\frac{1}{2}} \varphi^{\alpha(1-\lambda)}(x) \\
& \leq L_{6}(r+1)\left(\frac{n}{\varphi^{2}(x)}\right)^{r / 2} \varphi^{\alpha(1-\lambda)}(x)\|f\|_{0} .
\end{aligned}
$$

Hence, using $\delta_{n}(x) \sim \varphi(x)$ we obtain (3.1) for $x \in E_{n}$. We complete our proof by using (4.1) and (4.2). 
404 Shunsheng GUO, SHUJIE YUE, CUIXIANG LI, GE YANG AND YIGUO SUN

Proof of (3.4). We recall that [5]

$$
\left|B_{n}^{(r)}(f, x)\right| \leq n^{r} \sum_{k=0}^{n-r}\left|\vec{\Delta}_{\frac{1}{n}}^{r} f\left(\frac{k}{n}\right)\right| p_{n-r, k}(x) .
$$

For $0<k<n-r$, by [5, p. 155],

$$
\frac{k}{n}\left(1-\frac{k}{n}\right) \leq R\left(\frac{k}{n}+y\right)\left(1-\frac{k}{n}-y\right), \quad 0<y<\frac{r}{n},
$$

therefore $\delta_{n}\left(\frac{k}{n}\right) \leq R_{1} \delta_{n}\left(\frac{k}{n}+y\right)$. Hence we have, as in [5],

$$
\begin{aligned}
\left|\vec{\Delta}_{\frac{1}{n}}^{r} f\left(\frac{k}{n}\right)\right| & \leq R_{2} n^{-r+1} \int_{0}^{\frac{r}{n}}\left|f^{(r)}\left(\frac{k}{n}+u\right)\right| d u \\
& \leq R_{2} n^{-r+1}\|f\|_{r} \int_{0}^{\frac{r}{n}} \delta_{n}^{-r+\alpha(1-\lambda)}\left(\frac{k}{n}+u\right) d u \\
& \leq R_{2} n^{-r+1}\|f\|_{r} \frac{r}{n} \delta_{n}^{-r+\alpha(1-\lambda)}\left(\frac{k}{n}\right) .
\end{aligned}
$$

For $k=0$, note that $u \in\left(0, \frac{r}{n}\right)$ implies $\delta_{n}(u) \sim \frac{1}{\sqrt{n}}$. Thus, we have

$$
\begin{aligned}
\left|\vec{\Delta}_{\frac{1}{n}}^{r} f(0)\right| & \leq R_{3} \int_{0}^{\frac{r}{n}} u^{r-1}\left|f^{(r)}(u)\right| d u \\
& \leq R_{3}\|f\|_{r} \int_{0}^{\frac{r}{n}} u^{r-1} \delta_{n}^{-r+\alpha(1-\lambda)}(u) d u \\
& \leq R_{4} n^{-(r+\alpha(1-\lambda)) / 2}\|f\|_{r} .
\end{aligned}
$$

Similarly for $k=n-r$ we have

$$
\left|\vec{\Delta}_{\frac{1}{n}}^{r} f\left(\frac{n-r}{n}\right)\right| \leq R_{5} n^{-(r+\alpha(1-\lambda)) / 2}\|f\|_{r} .
$$

From (4.3)-(4.6) we get, with $\delta_{n}\left(\frac{k}{n}\right) \sim \delta_{n}\left(\frac{k+1}{n-r+2}\right)$,

$$
\begin{aligned}
& \left|\delta_{n}^{r+\alpha(\lambda-1)}(x) B_{n}^{(r)}(f, x)\right| \\
& \leq R_{6} n^{r}\|f\|_{r} \delta_{n}^{r+\alpha(\lambda-1)}(x)\left[n^{\frac{-r+\alpha(\lambda-1)}{2}}\left(p_{n-r, 0}(x)+p_{n-r, n-r}(x)\right)\right. \\
& \left.\quad+\sum_{k=1}^{n-r-1} n^{-r} \delta_{n}^{-r+\alpha(\lambda-1)}\left(\frac{k}{n}\right) p_{n-r, k}(x)\right] .
\end{aligned}
$$

By a simple computation, it is easy to get (cf. [2])

$$
\sum_{k=1}^{n-r-1}\left(\frac{n}{k}\right)^{r} p_{n-r, k}(x) \leq C \frac{1}{x^{r}}
$$

and

$$
\sum_{k=1}^{n-r-1}\left(\frac{n}{n-k}\right)^{r} p_{n-r, k}(x) \leq C \frac{1}{(1-x)^{r}}
$$


Hence

$$
\begin{aligned}
& \sum_{k=1}^{n-r-1} \varphi^{-2 r}\left(\frac{k}{n}\right) p_{n-r, k}(x) \\
& \quad \leq 2^{r} \sum_{k=1}^{n-r-1}\left(\left(\frac{n}{k}\right)^{r}+\left(\frac{n}{n-k}\right)^{r}\right) p_{n-r, k}(x) \leq C_{1} \varphi^{-2 r}(x) .
\end{aligned}
$$

Note that $\delta_{n}\left(\frac{k}{n}\right) \sim \max \left\{\varphi\left(\frac{k}{n}\right), \frac{1}{\sqrt{n}}\right\}$, and that by (4.7) and (4.8) we have

$$
\begin{aligned}
& \left|\delta_{n}^{r+\alpha(\lambda-1)}(x) B_{n}^{(r)}(f, x)\right| \\
& \leq R_{6}\|f\|_{r} \delta_{n}^{r+\alpha(\lambda-1)}(x)\left[n^{r}\left(p_{n-r, 0}(x)+p_{n-r, n-r}(x)\right)\right. \\
& \left.+\sum_{k=1}^{n-r-1} \delta_{n}^{-2 r}\left(\frac{k}{n}\right) p_{n-r, k}(x)\right]^{\frac{r-\alpha(1-\lambda)}{2 r}} \\
& \leq R_{7}\|f\|_{r} \delta_{n}^{r+\alpha(\lambda-1)}(x)\left(\min \left\{n^{r}, \sum_{k=1}^{n-r-1} \varphi^{-2 r}\left(\frac{k}{n}\right) p_{n-r, k}(x)\right\}\right)^{\frac{r-\alpha(1-\lambda)}{2 r}} \\
& \leq R_{8}\|f\|_{r} \delta_{n}^{r+\alpha(\lambda-1)}(x) \delta_{n}^{-r+\alpha(1-\lambda)}(x)=R_{8}\|f\|_{r} .
\end{aligned}
$$

Now we have proved the inequality (3.4). This finishes the proof of Lemma 3.1 .

Proof of Lemma 3.2. It is known that, for $0<t<\frac{1}{8 r}$, $\frac{r t}{2} \leq x \leq 1-\frac{r t}{2}$ (cf. [7]),

$$
\int_{-\frac{t}{2}}^{\frac{t}{2}} \cdots \int_{-\frac{t}{2}}^{\frac{t}{2}} \varphi^{-r}\left(x+u_{1}+\cdots+u_{r}\right) d u_{1} \cdots d u_{r} \leq C t^{r} \varphi^{-r}(x) .
$$

Using this and Hölder's inequality we obtain

$$
\begin{aligned}
& \int_{-\frac{t}{2}}^{\frac{t}{2}} \cdots \int_{-\frac{t}{2}}^{\frac{t}{2}} \delta_{n}^{-r+\alpha(1-\lambda)}\left(x+u_{1}+\cdots+u_{r}\right) d u_{1} \cdots d u_{r} \\
& \leq C_{1}\left(\int_{-\frac{t}{2}}^{\frac{t}{2}}\right. \\
& \left.\quad \ldots \int_{-\frac{t}{2}}^{\frac{t}{2}} \min \left\{\varphi^{-r}\left(x+u_{1}+\cdots+u_{r}\right), n^{\frac{r}{2}}\right\} d u_{1} \cdots d u_{r}\right)^{\frac{r-\alpha(1-\lambda)}{r}} t^{\alpha(1-\lambda)} \\
& \leq C_{2}\left(t^{r} \min \left\{\varphi^{-r}(x), n^{\frac{r}{2}}\right\}\right)^{\frac{r-\alpha(1-\lambda)}{r}} t^{\alpha(1-\lambda)} \\
& \leq C_{3} t^{r} \delta_{n}^{-r+\alpha(1-\lambda)}(x),
\end{aligned}
$$

which is the stated result.

\section{REFERENCES}

[1] H. Berens and G. G. Lorentz, Inverse theorems for Bernstein polynomials, Indiana Univ. Math. J. 21 (1972), 693-708. 
406 SHUNSHENG GUO, SHUJIE YUE, CUIXIANG LI, GE YANG AND YIGUO SUN

[2] Z. Ditzian, A Global inverse theorem for combinations of Bernstein polynomials, J. Approx. Th. 26 (1979), 277-292.

[3] Z. Ditzian, Direct estimate for Bernstein polynomials, J. Approx. Th. 79 (1994), 165166.

[4] Z. Ditzian and K. Ivanov, Bernstein-type operators and their derivatives, J. Approx. Th. 56 (1989), 72-90.

[5] Z. Ditzian and V. Totik, Moduli of Smoothness, Springer-Verlag, Berlin-New York, 1987.

[6] S. Guo and C. Li, Inverse estimate for Bernstein polynomials, to appear.

[7] L. Xie, On the Linear Combination of Bernstein Polynomials, J. Math. Res. Expos. 15 (1995), 577-582 (in Chinese).

DEPARTMENT OF MATHEMATiCS

HeBei TEACHER's UNIVERSITY

SHIJIAZHUANG 050016

People's Republic of China 


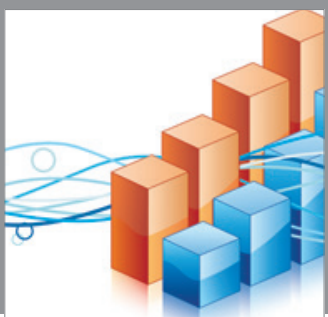

Advances in

Operations Research

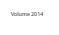

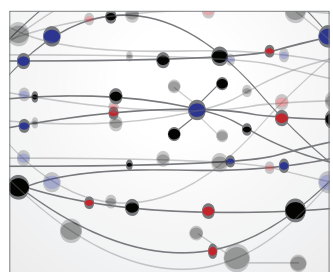

\section{The Scientific} World Journal
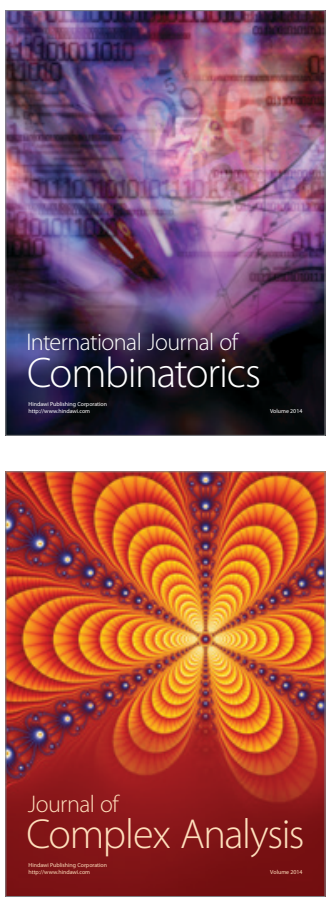

International Journal of

Mathematics and

Mathematical

Sciences
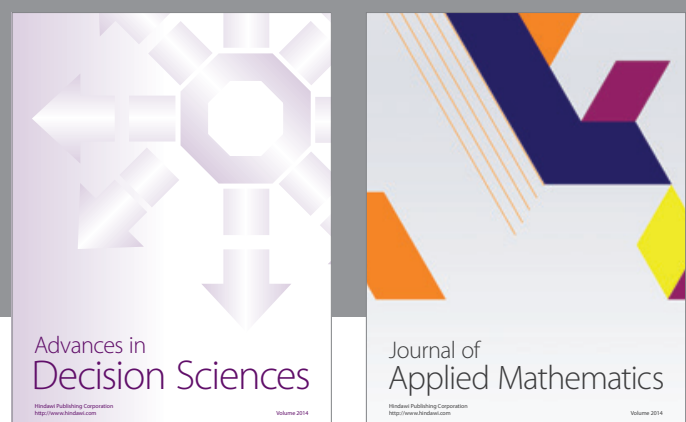

Journal of

Applied Mathematics
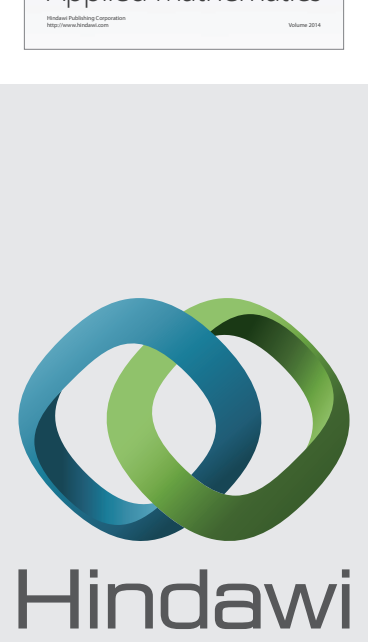

Submit your manuscripts at http://www.hindawi.com
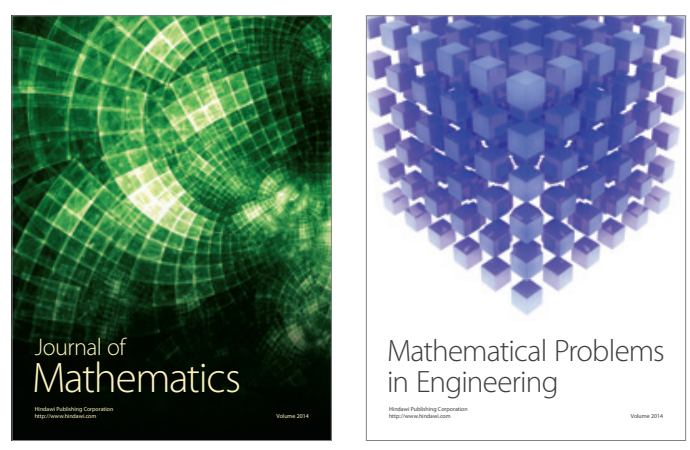

Mathematical Problems in Engineering
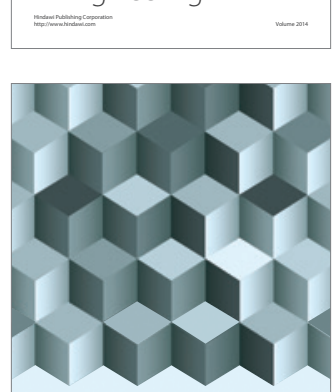

Journal of

Function Spaces
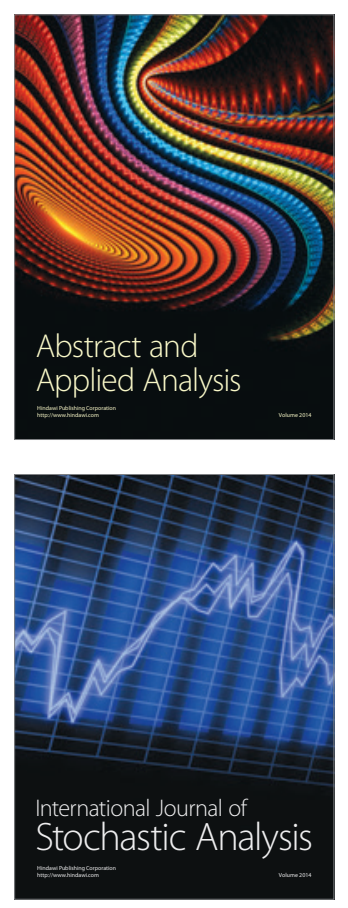

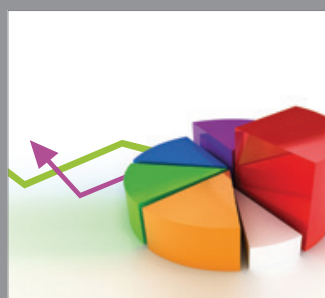

ournal of

Probability and Statistics

Promensencen
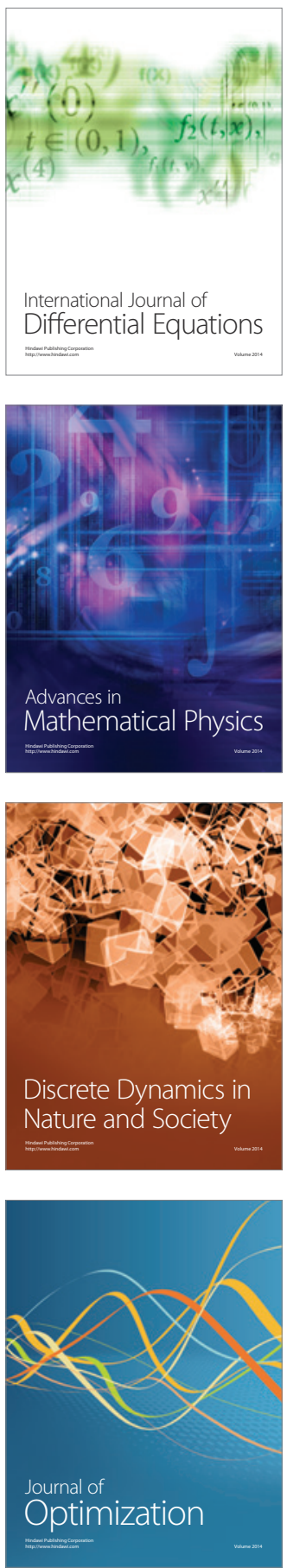